\title{
MOBILE ROBOT FOR EXPLORATION AND MANIPULATION Inside a Nuclear PoWer Plant
}

\author{
Niklas Ritzberger, Simon Emenshuber, Gerald Schubert, \\ Florian Voglsinger, Wilfried Kubinger \& Florian Fuchslocher
}
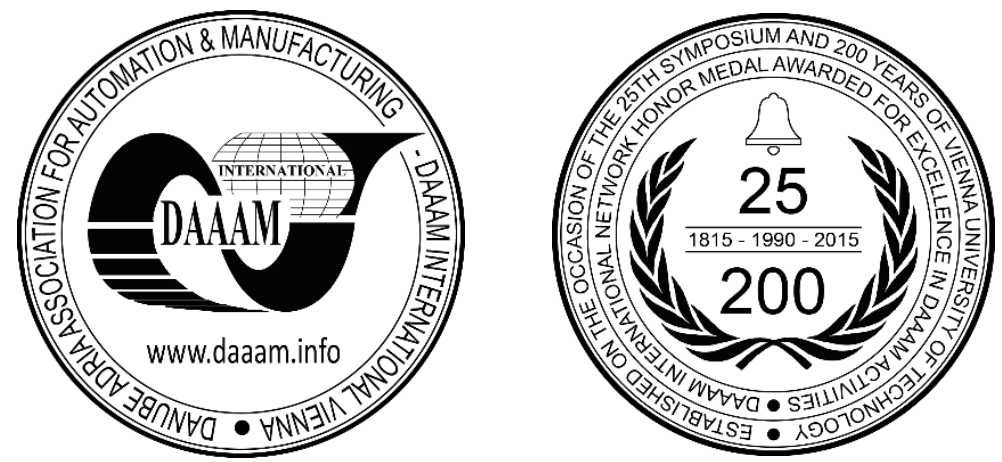

This Publication has to be referred as: Ritzberger, N[iklas]; Emenshuber, S[imon]; Schubert, G[erald]; Voglsinger, F[lorian]; Kubinger, W[ilfried] \& Fuchslocher, F[lorian] (2017). Mobile Robot for Exploration and Manipulation Inside a Nuclear Power Plant, Proceedings of the 28th DAAAM International Symposium, pp.0093-100, B. Katalinic (Ed.), Published by DAAAM International, ISBN 978-3-902734-11-2, ISSN 1726-9679, Vienna, Austria

DOI: $10.2507 / 28$ th.daaam.proceedings.012

\begin{abstract}
It is not easy to handle a nuclear accident in a nuclear power plant and keep the damage as little as possible. To alleviate the accident of Chernobyl, personnel were harmed. In case of Fukushima people were also sent into dangerous areas in the power plant. The first European Robotics Hackathon (EnRicH) in June 2017 was a robotics competition inside of a nuclear power plant in Austria, which was never put into operation. Creating a nuclear heat map within a two-dimensional map, a three-dimensional map and the manipulation of nuclear sources were the main tasks of the competition. A mobile robot called taurob tracker from the taurob $\mathrm{GmbH}$ was equipped with a SICK TIM551 laser scanner, a Kinect for XBoxOne, a nuclear sensor, cameras and a gripper. The 2D-mapping was accurate if enough features were recognized within an area of maximum 10 meters around the robot. With the Kinect a coloured point cloud was created. Successfully gripping a single nuclear source took about one minute. With the described robotic system, the given tasks of EnRicH 2017 have been successfully completed.
\end{abstract}

Keywords: EnRicH; mobile robot; mapping; ROS; teleoperation

\section{Introduction}

All over the world nuclear power plants are used to provide energy for industry, cities and peoples private consumption. Producing such type of energy can be very dangerous for the area around the power plant. It is not only that contaminated water could escape out of the power plant. The one of the worst cases is a meltdown of a reactor, like the Chernobyl or Fukushima disaster. The emergency workers had been harmed and died from the acute radiation syndrome. The area around the power plant is also still contaminated. To avoid humans getting harmed during the emergency work, robots will do that until it is save enough to enter the area. For this a mobile robotic system is needed. It must be a teleoperated system so the operator can stay in a save area. Additionally it should create a map, do measurements and is able to interact with his environment, for example with a gripper. 


\subsection{Motivation}

To evaluate the state of art for such robotic systems, European Robotics in cooperation with Fraunhofer FKIE, EnergySupply-Lower-Austria (EVN) and the Austrian Armaments and Defence Technology Agency (ADTA), initiate the first European Robotics Hackathon (EnRicH) in June 2017. It is a type of competition which took place inside of the nuclear power plant in Austria which was never put into operation. There have been three tasks to fulfil. The first was to create a 3D-map of the scenario. Create a Radiation Map where sources have to be detected and marked inside the map. The Last task was to detect and pick up radiation sources and put them into a bin [3].

\subsection{Problem description}

A Chain-Driven robot with a four-axis arm with 4 degrees of freedom (DOF) has to be equipped with the adequate sensors and actors. So a gripper has to be developed and sensors have to be chosen to create the maps. To teleoperate the robot also a visual system has to be mounted. An autonomous driving mode shall be implemented which navigates the system autonomous through the map.

\subsection{State of the art}

To run such a robotic systems the popular Robot Operating System (ROS) is used. It provides a bunch of driver for sensors and actors but also functions for teleoperation, mapping, navigation and a lot more. ROS can also run across multiple machines, which is needed if the robot has a build in embedded pc and the operation is done with the operator pc. For example the map will be calculated on the embedded computer because it provides more performance and then it will be shown on the operator's pc so an orientation is possible.

Taurob provides several ROS packages. These packages enable the control of the robot and the arm. The body of the robot can be controlled ether via the "teleop_twist_keyboard" or the "teleop_twist_joy" package. In both cases the key bindings can be set within a ".yaml" file. The key difference is as the names suggest that the "teleop_twist_keyboard" package enables the control via a keyboard and the "teleop_twist_joy" package via a controller. The used controller can be set within the "yaml” file. The arm can be controlled via the standard “joint_state_publisher" package. This reads the parameter of the robot and generates a graphic user interface (GUI). The axis can be controlled via sliders. Furthermore, two buttons are generated. The first button "Centering" centres all axis. The second button "Randomize" drives each axis to a random position. To note is, that all axis are moved at the same time. The reason for this behaviour is, that the values are first written into a variable and this variable is afterwards posted into the ROS topic.

The manipulator, which is mounted on the housing o the Taurob Tracker, is a robot arm with 4 degrees of freedom. The total reach is $140 \mathrm{~cm}$ and the maximum payload $5 \mathrm{~kg}$. The robot arm can be used to manipulate objects or open doors. In order to do this, different end effectors are necessary, which do not exist yet. The end effector is the device that achieves an effect and is located at the furthest point of a kinematic chain. In this work, a gripper is developed as an end effector, which is attached to the end of the robotic arm by the Taurob and serves as an interface between the robot and the object to be manipulated.

To find the objects that have to be manipulated and to fulfil the tasks of the challenge, a method of orientation for the robot or its operator in the unknown environment has to be found. This is accomplished by using a method of machine vision to create two and three dimensional maps. An overview of the machine vision technologies is given in [12]. Multiple of these technologies are also already described in use cases similar to the ones at the EnRicH 2017 [2]. Approaches the task with stereo vision and inertial measurements to determine the position of the robot and its surroundings. Another considered method was the use of multiple laser scanners, similar to [10]. In this work the use of a time of flight system, namely the Kinect for X-Box One was used to test if such a system can work on a very small budget.

Navigation of a Robot means the autonomous movement through an area without moving orders from the operator, only the target has to be defined. Navigation is possible through programmed behaviour or path planning. The combination of these two are the state of art. To realize the navigation a classification of the environment and the robotic system has to be done. It is necessary to know the operational area of the robot. Is it an unknown or known area, is it static or dynamic and is it structured or not. A static area means that no moving objects, like humans or vehicles are expected. Structured area for example are buildings or mostly differed by indoor and outdoor operation. The robot itself is defined as a non-holonomic robot, with differential drive. For a navigation a model of the environment is needed. Either there is already a model of the area or the robot has to create his own. The robot has to localize itself inside the model trough camera systems, laser scanner, triangulation or a combination of these systems. With the model and the localization the robot can calculate a path and follow it with the programmed behaviour.

\section{System Concept}

The used robot is a so called tracker from taurob GmbH. It is a Chain-Driven platform with a mounted 4-axis arm. It is designed to use it in difficult terrain and there is also an ATEX certificated variant to protect the robot against explosions. It is shown in figure 1. 


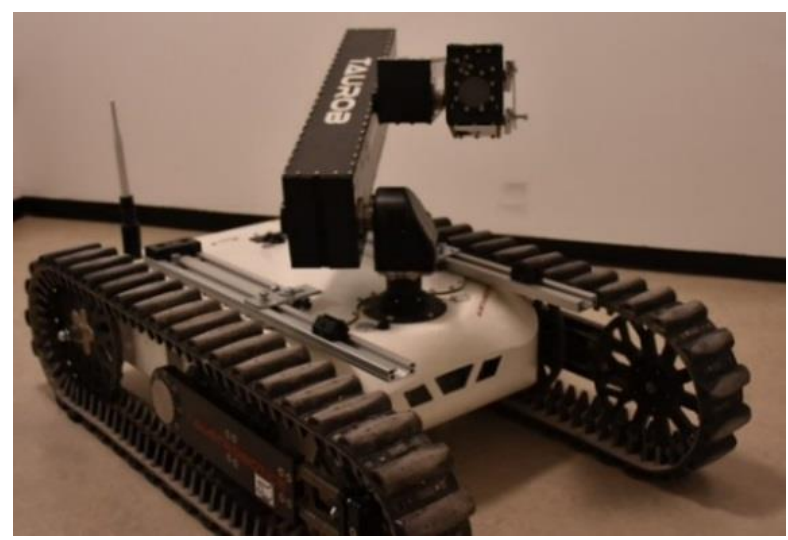

Fig. 1. Tracker robot without additional equipment

Inside of the white body are:

- electric motors

- WLAN Station

- one camera located at the glass in the middle

- another camera on the other side of the body

- LED lights at the decentred glasses

- the battery

- controller

A special feature of this robot is that the Chains can lift or lower partly to drive over stairs and obstacles. The arm has four degrees of freedom, turning around the $\mathrm{z}$-axis and $\mathrm{x}$-axis and movement along the $\mathrm{y}$-axis and the $\mathrm{z}$-axis. At the end of the arm there is a dummy sensor head [8].

\subsection{Additional Hardware}

To pass all the tasks sensors are needed and a gripper for manipulation. To get an overview over the environment of the robot two cameras got attached to the dummy sensor head at the arm. These help to drive through terrain with less space. A LED headlight was also installed to handle low light situations. For the 3D-mapping task the Microsoft Kinect was also attached to the head. The radioactive module SSM1+ which was provided was fixed on the arm and with a cable the sensor was fixed beneath the headlight. The At least the gripper got attached in front of the head. In figure 2 the head is shown but normally the Kinect is mounted on the side without the gripper.

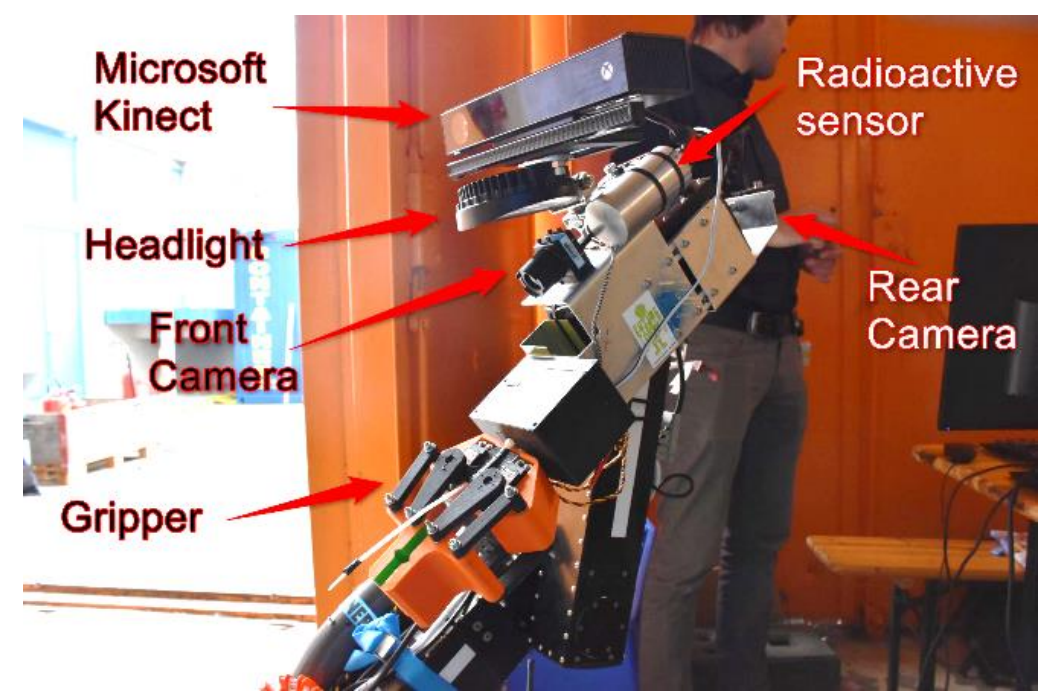

Fig. 2. Additional hardware at the end of the arm

On the Body an embedded pc is mounted. It controls the gripper, provides the data of the cameras and builds the 3Dmap. To get a 2D-map a LIDAR from SICK is mounted in front of the robot above the chains. With the range of ten meters and angular resolution of one degree an accurate map can be built. 


\subsection{Robot Operating System}

The Open Source framework 'Robot Operating System' (ROS) is the base to control the robot. Developed at the University of Stanford with the main targets:

- Peer-to-peer

- Tools-based

- Multi-lingual

- Thin

- Free and Open-Source

So ROS is a multi-host platform where a functions can work with all components. Functions can be written in different programming languages, the most common and best documented ones are Python and C++. Thin means to keep the function as simple and reusable as possible. Instead of running and building ROS functions in a large environment, a lot of small tools are doing this to keep the process as simple as possible [13].

\subsection{EnRicH 2017}

The EnRicH, which took place in the nuclear power plant (NPP) in Zwentendorf, Austria, is a competition which set the focus on detecting radiation sources, mapping and manipulation inside of a power plant. The testing area was the reactor hall on the last floor of the NPP. Around the fuel assembly changing basin and the fuel element cooling basin it was able act with the robot. The floor can be seen in the virtual tour on the Webpage of the NPP. The robot was brought up with a crane. Upstairs a Wireless Network was built up to stay in connection with the robot.

To create detailed 3D and 2D maps it was necessary to drive a complete round. The radiation sources should be measured and marked on a map. At one side of the basins radioactive sources and dummy sources where scattered and a marked bin stands beside. The challenge here was to differ between the real sources and the dummy ones. The next step was to pick up the real sources and throw them into the bin.

\subsection{Strategy}

Two valued runs were able to pass so the plan was to do the mapping and drive a whole round in the first run. The focus for the second run was on the manipulation task. Each run lasts 20 minutes. For the mapping challenge the Kinect was mounted without the gripper. One trial run was available which should be used to check if the time suffices to drive one time around the basins and create a sufficient map. The manipulation task has to be trained in the Pit lane with dummy sources.

\section{Methods}

With the chosen Hardware and ROS as base the needed parts got developed and implemented. The teleoperation, the gripper and the 3D-mapping was needed to complete all tasks. Additionally, an autonomous driving mode was implemented to drive back to start without orders from the operator.

\subsection{Teleoperation}

The teleoperation of the tracker can be split in three parts. These are the:

- Control of the body

- Control of the arm

- Control of the gripper

The control of the body two standard ROS-packages have been checked and compared. The "teleop_twist_keyboard" package enabled the control of the body through the keyboard. The speed of the robot can be changed via the push of a button, but the operator cannot vary the speed continuously [4]. The "teleop_twist joy" package enables the control via $\mathrm{X}$-Box controller. The controller can regulate the speed via an analogue stick [5]. Furthermore, a mode to halve the maximum speed of the robot was implemented by pressing the left bumper to get a more precise control.

The arm is controlled via a GUI. It allows the operator to individually position each axis. Furthermore, buttons with predetermined potions have been implemented. To achieve this the "joint_state_publisher" package has been used. It was altered to suit the robot and the given tasks.

The tree servomotors of the gripper were controlled via an Arduino micro. The Arduino was via the "Arduino" package integrated into the ROS-System. This enabled it to receive the servo positions via the UART bus. These positions were determined via a GUI. The distance between the jaws is displayed in millimetres in the GUI. To achieve this the servo angles are interpolated. 


\subsection{Gripper}

In the development of a gripper for mobile and service robotics, in contrast to automation technology, neither the properties of the object nor its position and orientation in space are known. After consultation with the organizers, a photo of the object that has to be manipulated was published. This is a metal rod with the approximate dimensions of $\mathrm{D}=12$ $\mathrm{mm} \times \mathrm{L}=110 \mathrm{~mm}$ and a mass of $0.1 \mathrm{~kg}$. The rod is hold with a force-fit in the $\mathrm{z}$-direction. The necessary gripping force can be calculated with equation 1 .

$$
F_{G}=m \cdot\left(a_{z}+g\right) \cdot \frac{\sin (\propto)}{2 \cdot \mu} \cdot S
$$

With an acceleration of $1 \mathrm{~m} / \mathrm{s}^{2}$, a coefficient of friction of 0.5 (equivalent to rubber on steel), an angle $\alpha$ of $45^{\circ}$ and a safety of 2, the gripping force is $1.53 \mathrm{~N}$. Now that the gripping force has been calculated, the necessary motor torque can be calculated. The equation 2 calculates the motor torque.

$$
M_{M}=F_{G} \cdot L
$$

In this case, it is important to ensure that the length $\mathrm{L}$ of a parallel jaw gripper with circular movement changes with opening and closing. Therefore, the maximum length that occurs when the gripper is closed must be used. With a length $\mathrm{L}$ of $0.09 \mathrm{~m}$, the resulting motor torque is $0.14 \mathrm{Nm}$. Now three Hitec HS-645MG servomotors have been selected, which provide a torque of $0.96 \mathrm{Nm}$.

The gripper was developed in the 3D CAD (Computer aided design) program SolidWorks 2016. During the construction of the individual parts, special attention was paid to the fact that the parts will be later produced with a Fused Deposition Modelling (FDM) 3D printer. The gripper essentially consists of two assemblies. The gripper itself and the rotary unit that allows to rotate the gripper by $90^{\circ}$.

\section{3. $3 D$ map}

For the creation of the three dimensional map, a "Kinect for X-Box One" was used. This version of the Kinect uses multiple cameras, a time-of-flight version for the depth information and a colour camera for colour information. This two separate information get then combined via an internal chip to create an RGB-D image. RGB-D stands for Red-GreenBlue-Depth and is basically a coloured point cloud. The algorithm that produces this is assisted by the third built in camera, which senses infra-red light and helps separating background and foreground. To make use of this information that is provided over the USB3.0 port of the Kinect, the open-source driver for ROS "libfreenect2" is used [15]. This library converts all the data of the Kinect to ROS standards and therefore further usage is enabled. The information provided by this can be directly used by several ROS programs, like the visualization RVIZ which is used for the 2Dmap and navigation. To generate 3D-maps the ROS package "rtabmap" was used. Like all the used ROS packages it is open-source. The program uses SIFT, Scale Invariant Feature Transform [9], and SURF, Speeded-Up Robust Features [1], for Feature detection in the RGB pictures. With this features it can determine which pixels are the same between multiple images and merge the respective point clouds. These merged clouds form the three dimensional map. The map can then be saved and viewed or loaded back into the program in a future mapping run and be continued or refined.

\subsection{D map and Autonomous Navigation}

The autonomous navigation was realized with a laser based Simultaneous Localization and Mapping (SLAM) and the algorithm called A Star Best first Search (A*) to navigate. SLAM is using the data of the laser scanner to create a map and locate the robot inside it. At Start-up of the system the robot creates a coordinate system with the centre point depending on his actual position. The algorithm matches the old and the new data from the laser scanner and a map is created when the robot is moving. The ROS-package 'Hector-SLAM' from university Darmstadt in Germany was implemented [6]. To create the heat map QGIS with the add-on 'heatmap' was used.

The algorithm A* calculates a path from goal to start. For that the map gets transformed to an occupancy grid. The fields of the grid can be occupied, free or unknown. If a field of the grid is partly covered by an object, it also counts as occupied [14]. The algorithm calculates always the fields around the actual field starting with the goal point. Each field get three values, the costs ' $\mathrm{g}(\mathrm{n})$ ' from goal to the actual field, the estimated costs ' $h(n)$ ' from the actual field to the start and the calculated costs ' $\mathrm{f}(\mathrm{n})$ ' for the complete way as it is shown in equation 3 .

$$
f(n)=g(n)+h(n)
$$

The algorithm is used by the ROS standard package for navigation [7]. The package was created during a project where a robot should navigate through an office. It got implemented in the ROS System and calibrated for the robot [11]. 


\section{Results}

All tasks had been passed successfully. During the first run the Kinect malfunctioned, so only the 2D-Map with the radiation mapping was created which can be seen in figure 4 . The second run was passed with mounted gripper and Kinect as it is shown in figure 1. Ten radioactive sources lied on the ground, five real and five dummy sources. Seven sources were lifted and five dummy sources were thrown into the bin by the operator. The way from start to the sources was mapped by the Kinect a picture of the 3D map is in figure 3.

\subsection{Teleoperation}

The two robot controls have been tested and their functions documented. The control via the keyboard allows the operator to change the maximum speed, but the robot can only drive at that speed. In case of the controller the maximum speed of the robot had to be set in the configuration files and the operator can drive at any speed between standing still and the maximum speed. To ensure the quality of the generated maps the maximum speed is capped at $40 \%$ of the maximum speed of the robot. This means the second, more precise mode runs at $20 \%$ speed.

Through tests the used GUI has been adapted to suit the robot and the challenges better. To prevent collision with the robot peripherals the end stops of the fist and the second axis had to be adjusted. The new end stop is for both axis is 0,2rad. Buttons with predetermined positions have been implemented. These are a button to centre each axis individually, a button for the camera position while driven and two buttons to position the gripper close to the floor. Furthermore, the arms reaction in case the connection between the operator and the robot is lost have been tested. The tests showed that the arm will always drive to the last received position and no further movement will be made until the connection is reestablished. Than the arm will drive to the current position on the GUI. The end stops of the gripper have been determined. They were set in a way that the jaws meet exactly in the middle of the gripper. When no object is grabbed, the jaws have a gape of about 0,5 millimeter between each other in the end position.

\subsection{Gripper}

To be able to test the parallel jaw gripper, steel pins were manufactured which are similar to the object EnRicH objects. The manipulating task of the EnRicH was trained at the military test ground Tritolwerk. The steel pins were placed vertically in a hole on a table. Now the gripper was used to pick up the steel pin and place it in a box. In order to find the exact position of the gripper in which the object can be gripped, a marked cable tie was attached to the gripper. The manipulation of the steel pin worked relatively well. But sometimes there were vibrations in the whole arm of the Taurob Tracker, which lead to shaking movements of the servomotors which are built into the gripper.

\section{3. $3 D$ map}

The map could be created and downloaded from the robot via Wi-Fi or a USB-stick. Static environments, like in the testing scenario at EnRicH could be mapped without to many problems as long as the operator of the robot drives carefully. The mapping is volatile if the robot makes some jerky movements and the operator has to prevent these as good as possible. Additionally, areas with lots of open space should be avoided, because the depth sensors of the Kinect only have a limited range of between three and five meters. Another weak point of the program used to generate is, that it assumes that there are no dynamic surroundings.

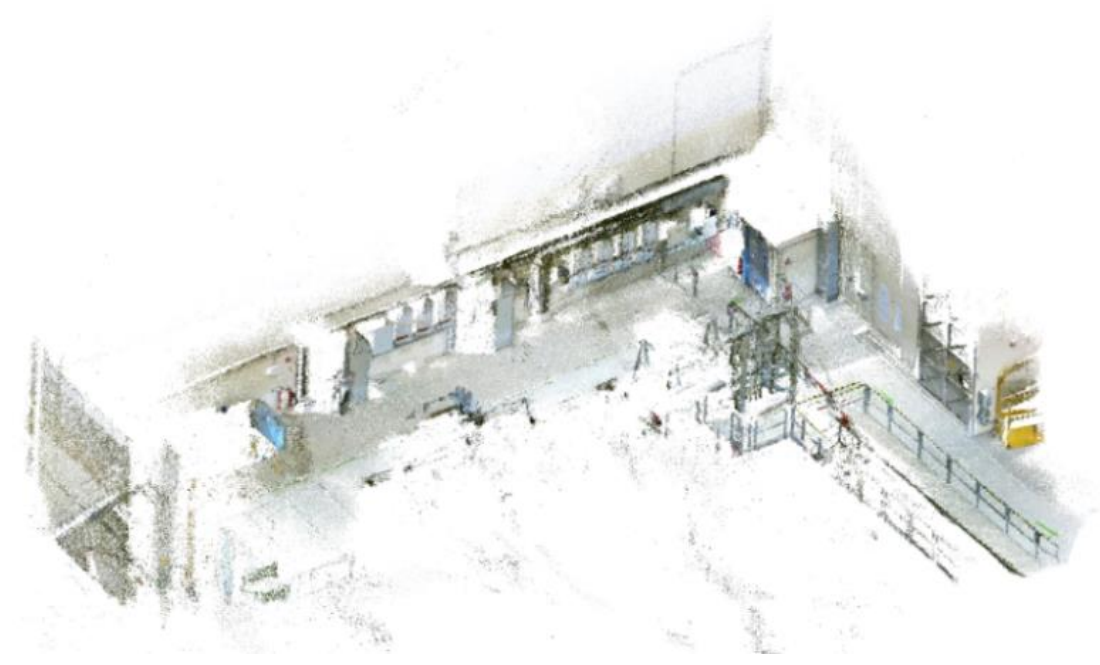

Fig. 3. 3D map from start to the hazard area 


\subsection{D map and Autonomous Navigation}

The 2D map was created with the SICK-Laser scanner. Because of the low range and the large distances a lot of problems came up especially when passing areas where only fence posts had been in range. In figure 4 the heat map is shown which was created during the first run. Two times the operator had driven too fast and too less reference points for the mapping tool had been in range so the map got a bend.

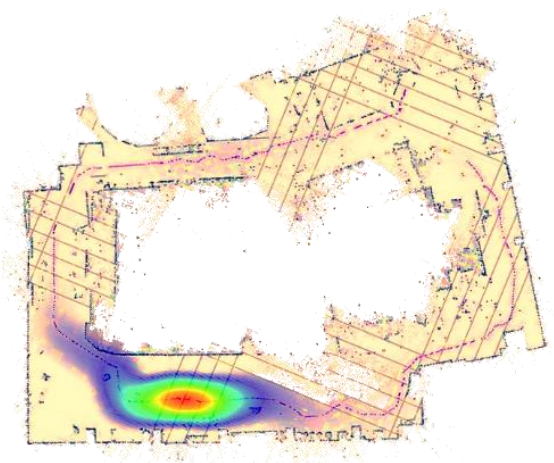

Fig. 4. 2D map of the reactor hall

The navigation wasn't used during the competition, because there have been too much complex objects like the fence posts and the danger to crash into a fence near the basins. Before the competition took place the navigation was tested with rooms and corridors. It worked after a lot of tests and calibrations.

\section{Discussion}

The setup of the robot was quite good. At the manipulation task the same amount of sources had been lifted compared to the winning team with automated manipulation functions. The predefined arm configurations made the whole process of grabbing a source very efficient. The Kinect worked well at the second run and the coloured point cloud is detailed and also small details can be identified.

\subsection{Teleoperation}

Through tests the handling via the X-Box controller proved to be more precise. Furthermore, the movement is smoother. This allowed for easier handling of objects and the map generation proofed to be more reliable. The GUI used to control the arm, proved to be reliable. The predetermined positions allowed the user to quickly move to often used arm configurations. To compensate a small offset between the two jaws, the endpoints of the jaws were adjusted. To ensure the jaws do not apply unnecessary force against each other while driving, the gap has been implemented.

The implementation of an inverse kinematic for the arm would be obvious. At the moment not all buttons on the controller are used. The implementation via the controller would assist the operator in controlling the robot.

\subsection{Gripper}

The stability of the 3D printed parallel jaw gripper was surprising. The vibrations occurred because the 0 -axis of the robot arm temporarily readjusts its position. Due to the long lever, this leads to a swinging movement of the gripper. The servo motors tried to compensate these vibrations which resulted in shaking movements of the gripper jaws. To solve this problem, the mounting plate was reworked for the EnRicH. It was shown that the shaking movements do not occur when the gripper holds the steel pin under force fit.

\section{3. $3 D$ map}

To improve the quality and reliability of the existing mapping solution, there are some parameters that could be tweaked. In the program there are options like the frame rate at which the map tries to refresh and how many common features are needed for two areas to count as identical. This tweaks come at the price of computation power. A more precise and reliable map generation therefore needs either a slower driving speed of the robot, which would have not been possible, given the time restriction of the challenge, or a more powerful PC mounted to the robot. More computing power comes with higher energy use and therefore shortens the time the robot can be operated between having to load its batteries. If the robot has to map a dynamic area, a new algorithm would be needed that can distinguish between moving and non-moving objects. 
Another way of optimizing the results could be to combine this method with other kinds of localization, like the also implemented 2D SLAM, to get a better estimation of the robots position and therefore an easier recognition of images that could show the same features. Another solution would be to use 3D laser scanners, which are more precise and can work on greater distances but the map would lose the coloured point clouds.

\subsection{D map and Autonomous Navigation}

The creation of the 2D map with the laser based SLAM is very efficient. The range of the laser scanner was a problem at the EnRicH. With a range above 20 meters the results would be much better, because in such an area more features can be recognized and the algorithm would be able to expand the map efficiently. Additionally the localization would be better which leads to better navigation results.

\section{Conclusion}

The first European Robotics Hackathon EnRicH 2017 was a competition where mobile robots had to solve tasks inside of a nuclear power plant with real radioactive sources. These tasks were to manipulation of objects, to create a 3D map of the scenario and to map the radioactive sources. The taurob tracker was equipped with an SICK TIM551 laser scanner, a Kinect for X-Box One, a nuclear sensor, cameras and a gripper. By the implementation of ROS the robot was controlled and all needed functions were available. The drive control was realized through an X-Box controller, the control of the arm and the gripper was done by a GUI on the operator computer. ROS packages had been used to create the 2D and 3D maps. All three tasks were successfully completed. The 2D map got a bend because of a driving mistake of the operator, but the map was still useable and the radioactive sources were correctly located. The Kinect had a failure at the first run. At the second run the Kinect mapped the way from the starting point to the hazard area where the manipulation task took place. Thanks to the Buttons in the arm control GUI the operator was fast enough to lift seven sources and throw five of them into the bin. With a laser scanner with more range a more precise map could be created easily because the operator would not have to be as carful while driving. The implementation of an inverse kinematic would make the arm control more intuitive because the operator can directly control the arm in three dimensions. This would mean the results would be much better. Also an Embedded PC with more power mounted on the robot would improve the quality of the 3D map.

\section{Acknowledgments}

This project has been funded by MA23 - City of Vienna within the Project Call 16-02 "Photonics: Foundations and industrial applications".

\section{References}

[1] Bay, H., et al, (2008). Surf: Speeded up robust features. In: Computer Vision and Image Understanding (CVIU). pp. $346-359$.

[2] Dias, J., Lobo, J., \& Queiroz, C. (2003). World feature detection and mapping using stereovision and inertial sensors. Robotics and Autonomous Systems, 44, 69-81. DOI: 10.1016/S0921-8890(03)00011-3

[3] http://enrich.european-robotics.eu/, (2016). European Robotics, The First European Robotics Hackathon, Accessed on: 30.08 .2017

[4] http://wiki.ros.org/teleop_twist_keyboard, (2016). Jay, G., teleop_twist_keyboard, Accessed on: 2017-06-02

[5] http://wiki.ros.org/teleop_twist_joy, (2016). Purvis, M., teleop_twist_joy, Accessed on: 2017-06-02

[6] http://wiki.ros.org/hector_mapping, (2012). Kohlbrecher S., hector_mapping, Accessed on: 30.08.2017

[7] http://wiki.ros.org/navigation, (2017). Marder-Eppstein, E., navigation, Accessed on: 01.09.2017

[8] http://taurob.com/produkte/ugv-taurob-tracker, (2016). Taurob GmbH, UGV - taurob tracker, Accessed on: 30.08.2017

[9] Lowe, D. G., (2004). Distinctive image features from scale-invariant keypoints. In: International Journal of Computer Vision. pp. $91-110$.

[10] Lepej, P., Lakota, M. \& Rakun, J., (2016). Simultaneous Localization, Mapping and Scene Reconstruction, Chapter 07 in DAAAM International Scientific Book 2016, pp.069-076, B. Katalinic (Ed.), Published by DAAAM International, ISBN 978-3-902734-09-9, ISSN 1726-9687, Vienna, Austria DOI: 10.2507/daaam.scibook.2016.07

[11] Marder-Eppstein E., et al,(2010). The office marathon: Robust navigation in an indoor office environment, International Conference on Robotics and Automation, in press

[12] Pérez, L. et al., (2016). Robot Guidance Using Machine Vision Techniques in Industrial Environments: A Comparative Review. Sensors, 16(3), p.335. Available at: http://dx.doi.org/10.3390/s16030335.

[13] Quigley, M., et al, (2009). Ros: an open-source robot operating system, ICRA Workshop on Open Source Software, in press

[14] Thrun, S., Burgard, W. \& Fox, D., (2006). Probabilistic Robotics, The MIT Press, ISBN 978-0-262-20162-9, Cambridge, US

[15] Xiang, L., et al, (2016). libfreenect2: Release 0.2. Available from: https://doi.org/10.5281/zenodo.50641 Accessed 2017-09-07 